\title{
EVALUATION OF THE CHANGES OF GAIT STABILITY ON THE TREADMILL DUE TO TREADMILL VELOCITY CHANGES BY MEANS OF TIME-FREQUENCY ANALYSIS
}

\author{
P. Wodarski ${ }^{*}$, J. Jurkojć ${ }^{* *}$, A. Bieniek ${ }^{* * *}$, R. Michnik ${ }^{\dagger}$, M. Gzik ${ }^{*}$, \\ M. Chrzan ${ }^{\dagger \dagger}$, M. Galązka ${ }^{\S}$, C. Herrera Ligero ${ }^{\S \S}$
}

\begin{abstract}
The frequency analysis enables the determination of changes of the COP trajectories during gait by means of detection of constantly repeated fragments. The time-frequency analysis was used in the conducted tests in order to assess the stability of gait on the treadmill. A group of 32 subjects participated in the tests at the age of 23(SD 1.8). The course of testing encompassed gait on the treadmill for $120 \mathrm{~s}$. Initial velocity equalled $V 1=3.2 \mathrm{~km} / \mathrm{h}$ for $60 \mathrm{~s}$, then the treadmill accelerated by $25 \%$ to $4 \mathrm{~km} / \mathrm{h}$ for subsequent $60 \mathrm{~s}$. On the basis of the recorded distribution of pressure exerted on the ground and a software programme dedicated to treadmills, the following kinematic values were determined: cadence, stride time, stride length, step width for each examined group of participants and the COP position for the ML and AP directions. Using Matlab software programme, the researchers examined time-frequency dependencies and defined time-frequency periodograms for the ML and AP directions of the COP motions. In order to conduct a quantitative assessment of results, PSD coefficients were determined. The results indicate statistically significant changes in kinematic parameters except for the step width. The COP trajectories show the appearance of additional components indicating the change of gait stability. The changes of a method of body balancing and at the same time a way of the COP displacement during gait were confirmed by the analysis of the harmonic amplitudes. The conclusions show that the time-frequency analysis made it possible to determine changes in stability during the whole investigation on the grounds of the COP measurements. This methodology may be used to assess progress of the therapy of patients with balance instability.
\end{abstract}

Keywords: gait, time-frequency analysis, COP, diagnosis

\section{Introduction}

Disorders of gait stability may be a symptom of neurological problems or problems with the motor system (Cikajlo, 2009, Joszko, 2018). Such disorders may be diagnosed on the basis of the measurements of the distribution of force values on the ground in time (Rosenblatt, 2010). It is possible to carry out measurements in a position of standing and during gait. The following kinematic parameters are often

\footnotetext{
* Piotr Wodarski PhD. Eng: Department of Biomechatronics, Faculty of Biomedical Engeenering, Silesian Univesity of Technology; Poland, piotr.wodarski@polsl.pl

** Jacek Jurkojc PhD. Eng: Department of Biomechatronics, Faculty of Biomedical Engeenering, Silesian Univesity of Technology; Poland, jacek.jurkojc@polsl.pl

*** Andrzej Bieniek MSc. Eng: Department of Biomechatronics, Faculty of Biomedical Engeenering, Silesian Univesity of Technology; Poland, andrzej.bieniek@polsl.pl

$\dagger \quad$ Robert Michnik PhD. DSc. Eng: Department of Biomechatronics, Faculty of Biomedical Engeenering, Silesian Univesity of Technology; Poland, robert.michnik@polsl.pl

\$ Prof. Marek Gzik, Professor PhD DSc. Eng.,: Department of Biomechatronics, Faculty of Biomedical Engeenering, Silesian Univesity of Technology; Poland, marek.gzik@polsl.pl

i† Miłosz Chrzan MSc. Eng: Department of Biomechatronics, Faculty of Biomedical Engeenering, Silesian Univesity of Technology; Poland; milosz.chrzan@polsl.pl

$\S$ Mariusz Gałązka MSc. Eng: Faculty of Biomedical Engeenering, Silesian Univesity of Technology; Poland, mariusgalazka@gmail.com

$\S \S$ Cristina Herrera Ligero, PhD.: Instituto de Biomecánica de Valencia (IBV), Valenciana, Spain; cristina.herrera@ibv.upv.es
} 
measured to assess gait: stride length, frequency of making strides, width of steps as well as location of the centre of pressure (COP) in subsequent moments of time (Menz, 2003, Krebs, 2002). Enrichment of classical methods of the kinematic parameters analysis with the frequency analysis and the timefrequency analysis brings several benefits (Dlugosz, 2018). For instance, it is possible to obtain mean values of the COP trajectories in any plane for unsynchronized measurements, which is not possible in the case of time (Michnik, 2016). It is particularly justified to use the frequency analysis and the timefrequency analysis in the cases where the investigated signal shows a feature of periodicity. The frequency analysis enables the determination of changes in the signal course by means of detection of constantly repeated fragments of the course. This is not possible while using the classical analysis of kinematic parameters (Jurkojć, 2017).

This research aims to evaluate changes in gait stability on the treadmill resulting from the impact of visual stimuli generated by the Virtual Reality Technology. The conducted investigations constitute the first stage of the research on the application of methods of time-frequency analyses for the evaluation of instability of gait performed at two different velocities. The conducted analyses and the developed methodology will be used to detect the impact of visual stimuli generated by the VRT and a 3D headset on the changes in gait stability.

The detailed aim of the conducted tests was the evaluation of the changes in the courses of the COP (centre of pressure) trajectories and at the same time the changes in the stability of gait on the treadmill due to treadmill velocity changes. The researchers performed the quantitative assessment of the changes of the courses of the COP trajectories in the medio-lateral (ML) plane and the anterior-posterior plane (AP) using the time-frequency analysis.

\section{Methods}

Participants: Participants: A group of 32 subjects took part in the tests (26 women and 6 men) at the age of 23 (SD 1.8), average height: $170 \mathrm{~cm}$ (SD 8.7), average weight: $63 \mathrm{~kg}$ (SD 15.2) and average BMI: 21.47 (SD 3.4). Each participant declared that they had not had any lower limbs injuries or suffered from any ailments connected with the motor system or balance instability.

Course of study: The course of the investigation included gait on the treadmill for $120 \mathrm{~s}$, initially at a velocity of V1 $=3.2 \mathrm{~km} / \mathrm{h}$ for $60 \mathrm{~s}$, next the treadmill accelerated by $25 \%$ to a speed of $4 \mathrm{~km} / \mathrm{h}$ for subsequent $60 \mathrm{~s}$.

Data collection: In the conducted tests, a WinFDM-T treadmill of the Zebris company was used to measure the distribution of pressure forces. Additionally, safety measures were applied in the form of a harness to protect the subjects from an accidental fall. One-hundred-and-twenty-second distributions of feet pressure on the ground were recorded for each participant.

Calculations: On the basis of the recorded distributions of pressure forces exerted on the ground and a special software programme dedicated to treadmills, the following kinematic values were determined: cadence, stride time, stride length and step width for each tested group. Mean values are presented in Fig. 1a. The applied software programme made it possible to export the COP trajectories in the ML and AP directions to the Matlab programme. An example of the COP trajectory in the ML direction is presented in Fig 1b. In order to examine time-frequency dependencies, the STFT calculations (Short-time Fourier transform, window $20 \mathrm{~s}$, shift $1 \mathrm{~s}$ ) were conducted in the Matlab programme. Next, time-frequency periodograms for the ML and AP directions of the COP motions were determined. The periodograms for each subject were standardized to their individual frequency of making strides for velocity V1 (such individual frequency was defined in the first stage of the investigations). Next, the periodograms were averaged. In the subsequent phase, in order to evaluate the amplitude of the COP deviations in the AP and ML directions, the PSD (power spectral density) profiles were determined for the frequency with the highest amplitudes. The way of plotting these profiles was presented in Fig. 2 and Fig 3.

\section{Results}

Figure 1a presents mean values of the measured kinematic parameters, such as: cadence, stride time, stride length and step width for the tested group of subjects, whereas figure $1 \mathrm{~b}$ shows an example of the COP trajectory in the ML direction. Figure 2 presents periodograms calculated for the COP displacements 
in the ML direction for each time moment, then it shows their top view and profiles for two selected frequencies. Figure 3 demonstrates periodograms calculated for the COP displacements in the ML direction for each time moment, then it shows their top view and profiles for two selected frequencies.

1a) cadence $[\mathrm{step} / \mathrm{min}]$

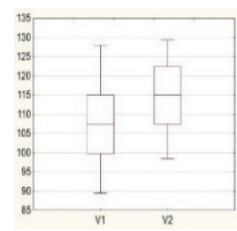

stride time $[\mathrm{s}]$

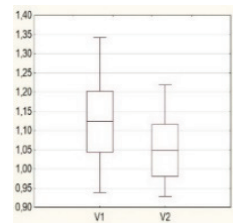

stride length $[\mathrm{cm}]$

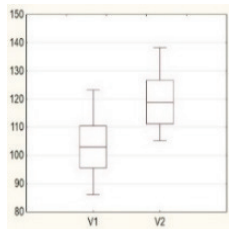

1b) step width $[\mathrm{cm}]$

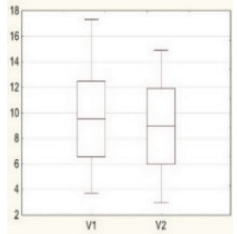
COP course - ML
$[\mathrm{mm}]$

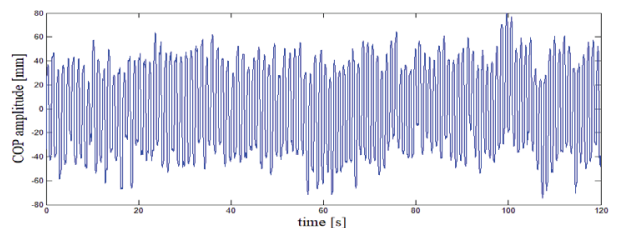

Fig. 1: A) Mean values of kinematic parameters measured for the tested group of subjects. Values with standard deviation as well as the range of values were marked in the chart; $B$ ) an example of the COP trajectory in the ML direction
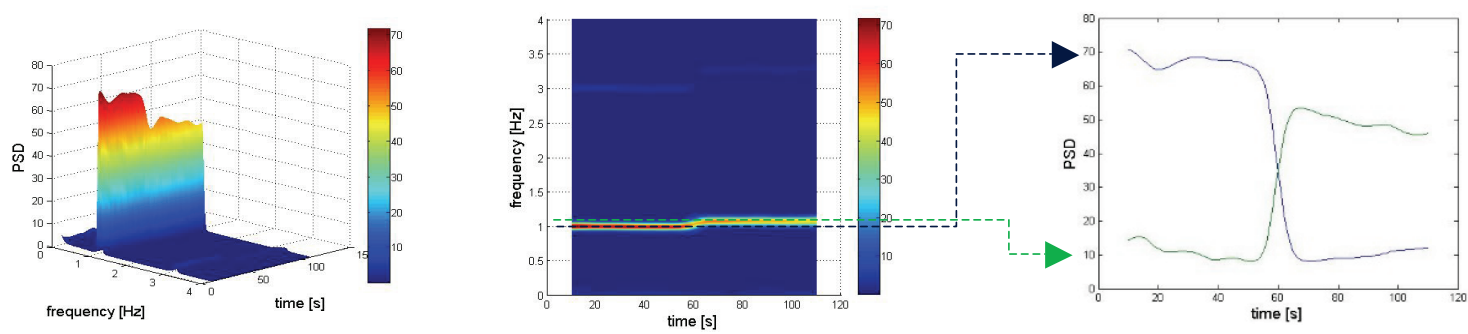

Fig. 2: Chart of periodograms for the COP displacements in the ML direction for subsequent time moments (a profile view and a top view) as well as two profiles for the frequency of the highest amplitudes
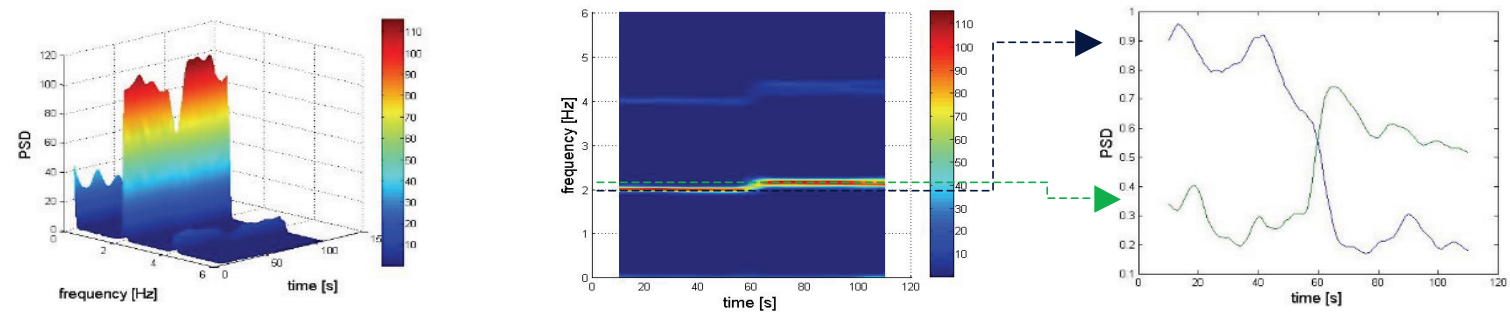

Fig. 3: Chart of periodograms for the COP displacements in the AP direction for subsequent time moments (a profile view and a top view) as well as two profiles for the frequency of the highest amplitudes

\section{Discussion}

The conducted calculations reveal a statistically significant change of the kinematic values measured during gait on the treadmill at velocity V1 and V2. The increase of the following parameters: cadence $(\mathrm{p}=0.00017)$ and stride length $(\mathrm{p}<0.00001)$ was observed for velocity V2, whereas the parameter of stride time $(p=0.00016)$ decreased. The value of the parameter of step width did not change due to the change of the treadmill velocity $(p=0.45)$. The increase of the frequency of making steps and the decrease of time of the gait cycle may reflect a subjective fall of the feeling of stability in subjects.

The STFT analysis of the COP trajectories in the ML and AP directions provides information on the method of carrying loads during gait on the treadmill at velocity V1 and V2. In Figures 2 and 3, averaged periodograms show a stable value of the main component of the COP which represents the frequency of making strides for the first $60 \mathrm{~s}$ of the test. This value amounts to $1 \mathrm{~Hz} / \mathrm{Hz}$ for the ML direction and 2 $\mathrm{Hz} / \mathrm{Hz}$ for the AP direction.

The displacement of the visible red line by around $60 \mathrm{~s}$ in the direction of higher frequencies reflects the change in frequency of making strides due to the change in the treadmill velocity. The chart also presents stripes of a smaller amplitude and frequency of $3 \mathrm{~Hz} / \mathrm{Hz}$ for the ML course and $4 \mathrm{~Hz} / \mathrm{Hz}$ for the AP course. They mean that the COP trajectories in the AP and ML directions have more harmonic components and even in the case of averaging for the whole group they do not have a sinusoidal trajectory. 
In the area of $60 \mathrm{~s}$ one may also observe the change of the higher harmonic frequencies proportionally to the components with the highest amplitude. The analysis of the time courses of the changes of the highest harmonic amplitudes, which were created as a result of the section of profiles from periodograms (in accordance with the described methodology; marked with blue and green arrows), makes it possible to define the moment of the change of frequency $-60 \mathrm{~s}$ (the intersection of the green and blue lines).

Higher values in the trajectories marked with the blue line for the AP direction during the first $60 \mathrm{~s}$ of the test in relation to the values marked with the green line indicating the time after the initial $60 \mathrm{~s}$ of motion - show a higher amplitude of the main harmonics, which reflects a longer stride in the first part of the test. This information is also confirmed by the analysis of the parameter of stride length. It is most interesting that this dependence is also visible for the ML direction. The highest harmonics for V1 in the ML direction has a higher value of the amplitude in the first part of the test (up to $60 \mathrm{~s}$ ) than in the second part of the investigation (after $60 \mathrm{~s}$ ). This means the fall of the amplitude of the COP trajectory in the ML direction, which is not confirmed by the analysis of the step width. The conducted analysis indicates a change in the way of carrying loads in the ML direction without any changes in the width of steps.

\section{Conclusions}

The time-frequency analysis of the COP trajectories during gait on the treadmill enables the determination of changes in the stability of gait at any section of time during the test. The analysis makes it possible to evaluate the way of moving the centre of pressure during the whole gait cycle. The conducted analyses of the COP locations are confirmed by the results of the calculations of parameters connected with the frequency of making steps and stride length. However, the results do not confirm the changes in the step width, which may mean a change in the method of gait or a change in the way of body balancing in the case of higher velocity. The developed methodology may be used to assess the impact of repeated external stimuli, such as those generated by the Virtual Reality Technology, on the change of gait stability in the ML and AP directions for people after stroke.

\section{Acknowledgments}

This work has been done under the project "Development of Innovative Training Contents Based on the Applicability of Virtual Reality in the Field of Stroke Rehabilitation", contract number: 2017-1-PL01KA202-038370. This project has been funded with support from the European Commission, Program Erasmus + . This communication reflects the views only of the authors, and the Commission cannot be held responsible for any use which may be made of the information contained therein.

\section{References}

Cikajlo I., Matjaćić Z. (2009) Advantages of visual reality technology in rehabilitation of people with neuromuscular disorders, Recent Advances in Biomedical Engineering, , ISBN 978-953-307-004-9, pp. 301-320

Długosz M., Wodarski P., Bieniek A., Chrzan M., Gzik M., Joszko k., Derejczyk J. (2018): Determination of the number and frequency of steps for gait with elbow crutches based on a crutch acceleration, Springer, Innovations in Biomedical Engineering, Advances in Inteligent System and Computing 623, ISSN 2194-5357, chapter 26, pp. 245-253

Joszko K., Gzik M., Wolański W., Gzik-Zroska B., Kawlewska E. (2018) Biomechanical evaluation of human lumbar spine in spondylolisthesis, Journal of Applied Biomedicine16, pp. 51-58

Jurkojć J., Wodarski P., Bieniek A., Gzik M., Michnik R. (2017) Influence of changing frequency and various sceneries on stabilometric parameters and on the effect of adaptation in an immersive 3D virtual environment, Acta of Bioengineering and Biomechanics, DOI assigned: 10.5277/ABB-00784-2016-02, vol. 19, No. 3, pp. $129-137$

Krebs D.E., Goldvasser D., Lockert J.D., Portney L.G., Gill-Body K.M. (2002) Is base of support greater in unsteady gait? Phys Ther, 82, pp. 138-47

Menz H.B., Lord S.R., Fitzpatrick R.C. (2003) Age-related differences in walking stability. Age Ageing, 32, pp. 137-142

Michnik R. Jurkojć J., Wodarski P., Gzik M., Jochymczyk-Woźniak K., Bieniek A. (2016) The influence of frequency of visual disorders on stabilographic parameters, Acta of Bioengineering and Biomechanics DOI: 10.5277/ABB-00201-2014-04, vol. 18, No. 1, pp. 25-33

Rosenblatt, N.J., Grabiner, M.D. (2010) Measures of frontal plane stability during treadmill and overground walking. Gait Posture 31, pp. 380-384 